\title{
A METANET ARCHITECTURE FOR END-TO-END QUALITY OF SERVICE (QOS) OVER DISPARATE NETWORKS
}

\author{
S. D. Jones and I. J. Wang \\ The Johns Hopkins University Applied Physics Laboratory \\ 11100 Johns Hopkins Rd. \\ Laurel, MD 20723 \\ steven.jones, i-jeng.wang@jhuapl.edu \\ E. K. P. Chong and H. J. Siegel \\ Colorado State University \\ Electrical and Computer Engineering Dept. \\ Ft. Collins, CO 80523 \\ echong,hj@engr.colostate.edu
}

\begin{abstract}
In this work we investigate an architecture for resource provisioning and route selection over multiple disparate networks. We have developed a layered hierarchy of inter-networking and intra-networking levels of management called a MetaNet. Management at the inter-network level is conducted in a manner that models each network abstractly. At the intra-network level management accounts for the detailed implementation and organizational characteristics of each network. The paper explores how to employ these layers to cooperatively satisfy end-to-end requests for connectivity with an associated QoS. In addition, we explore negotiation between the inter-and intra-network levels of management and brokering of QoS by the internetwork level among the intra-network level managers.
\end{abstract}

\section{ARCHITECTURE}

The Defense Research Projects Agency (DARPA) Information Systems Office (ISO) established the Agile Information Control Environment (AICE) Program [1] to develop a theoretical foundation and enabling technology to dynamically manage and control information flows across a distributed and heterogeneous network infrastructure that consists of both military and commercial networks. The ultimate objective of the AICE program was to realize information control in a way that is faster, more efficient, and more precise than is currently realized. A four-layer functional architecture for AICE is depicted in Figure 1. Brief descriptions of the function performed by each layer follow:

The Physical Network Layer: This layer will consist of many independently owned and operated tactical and commercial networks that, in general, provide routing services and unique QoS capabilities. These are the communication resources via which the information flows will be conveyed.

The MetaNet Layer: Future military information operations will employ a variety of commercial and DoD enterprise networks. Information will be disseminated based upon the QoS required by the user and the requisite networks will be selected on a message basis for their ability to achieve the QoS. The MetaNet is the system that facilitates the QoS based routing via this collection of networks. Four aspects of the MetaNet layer include: inserting QoS-like capabilities into existing tactical networks to enable dynamic reallocation of network resources [2], negotiating service requests as an intermediary between the user and individual networks, providing end-to-end QoS solutions within a time constraint, and maintaining negotiated end-to-end QoS by dynamically re-routing or renegotiating service.

The Adaptive Information Control (AIC) Layer: This layer provides global "content-aware" dynamic information flow control, employing the services of the MetaNet layer to do so. Features of the AIC layer include: partitioning of information flows among available logical channels, globally optimizing allocation to achieve military users information flow priorities [3], and reallocating resources when necessary due to network QoS degradation.

The Information Policy Management (IPM) Layer: This layer has three primary functions: providing users the capability to visualize the impacts of their information

This work was supported by the Defense Advanced Research

Projects Agency (DARPA) under the Agile Information Control

Environment Program, contract number DABT63-99-C-0010. 
control policies, relating information policy management to military operations, and aiding in the synthesis of effective information control policies.

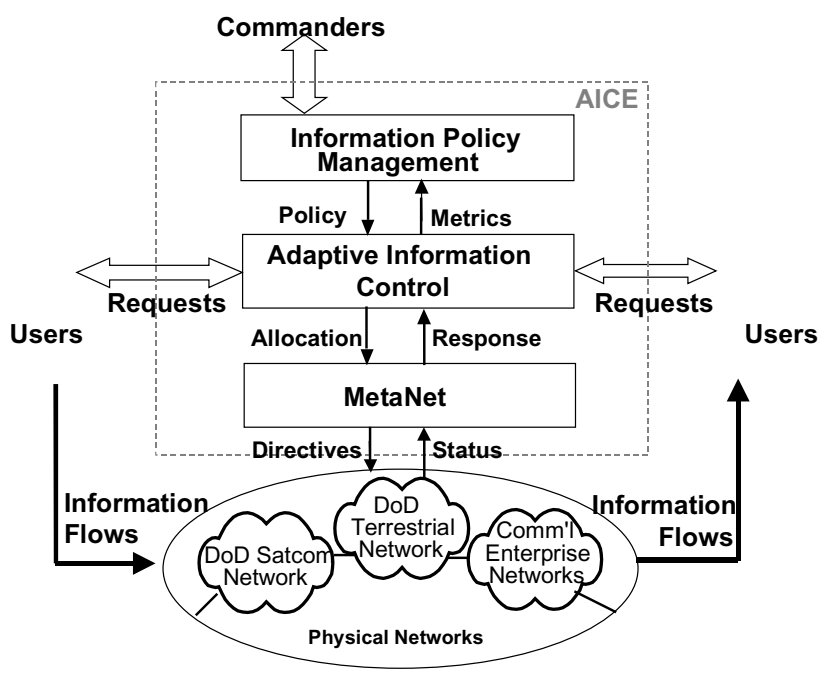

Figure 1 - AICE Architecture

\section{METANET}

The main task of the MetaNet layer is to negotiate and coordinate the setup of end-to-end QoS across multiple QoS-dissimilar networks and to perform inter-network routing. The MetaNet will provide a set of end-to-end logical channels (each logical channel may span several physical networks) with specific guaranteed QoS to the AIC layer for information flow control. The MetaNet will also monitor and maintain the QoS status of each established logical channel and report any change in channel QoS to the AIC layer for appropriate responses or necessary information channel reconfigurations.

The initial MetaNet interface to two networks has been developed: the DoD extremely high frequency (EHF) Satcom Network and a commercial asynchronous transfer mode (ATM) network. In each of these networks circuits have been established with specified QoS in a testbed environment [4]. The MetaNet concept will allow more efficient use of available networks for tactical communications applications than is presently realized by eliminating the use of "stove-pipes" in which information is always transported via a fixed medium.

The concept of operations for the MetaNet includes interfaces to a higher AIC layer, conveying user requirements, and subordinate tactical communications networks. The AIC will pass user requests for communications access to the MetaNet along with a set of QoS requirements. The MetaNet will assess the available connectivity and QoS through negotiation with and analysis of performance and capacity of the subordinate networks. Resulting solutions will optionally be returned to the AIC, where connectivity decisions are made. The AIC will then inform the MetaNet and the MetaNet will direct resource allocation in the chosen communications system to establish connectivity.

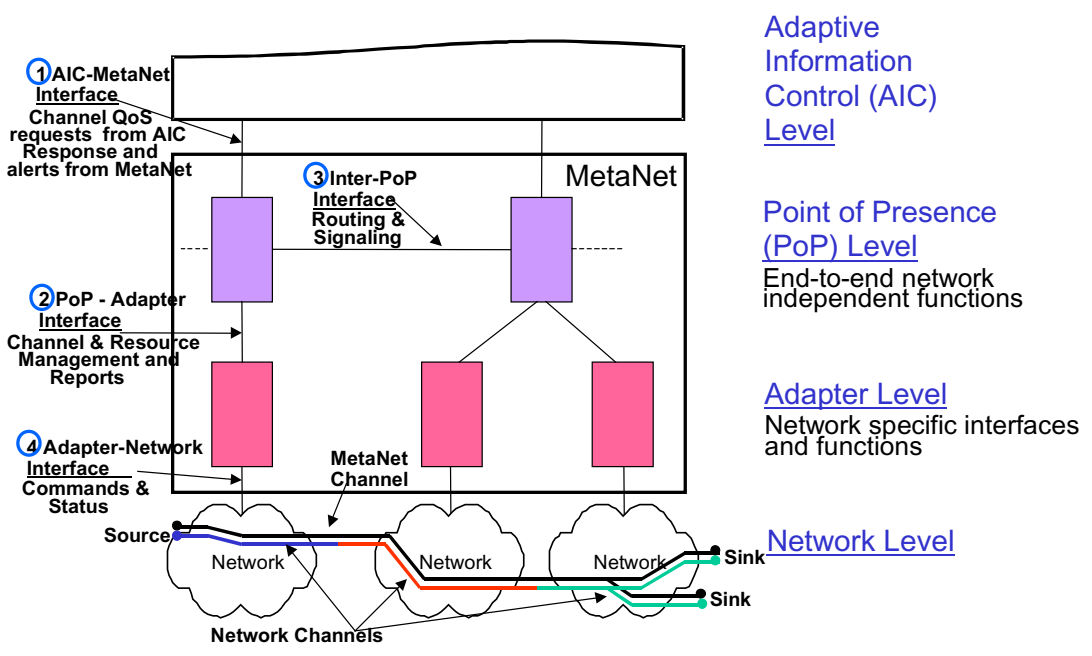

Figure 2-MetaNet Architecture 


\section{METANET ELEMENTS AND INTERFACES}

\subsection{Elements}

The MetaNet is composed of elements of two types as shown in Figure 2: the MetaNet Point of Presence (PoP) and the adapter. The PoP performs inter-network functions while maintaining interfaces to the AIC, to other PoPs, and to one or more adapters that it manages. The adapter controls a physical network, either through its network management element or through the nodes of the network. The adapter interfaces with a PoP and with the network.

The MetaNet PoP represents the interface point for external (i.e., AIC) communication with the MetaNet. Hence the term PoP is used in this context. A distinction can be made however, in that at the MetaNet PoP the exchange is on a control domain rather than on the information flow domain. In the MetaNet architecture, the information flows across the networks being controlled by MetaNet rather than through the MetaNet.

All PoPs are of the same design, that is, the software is identical in each PoP. Adapters, however, are designed to control a specific network technology and thus are unique for each network. The value of this approach is in the ability to simply replicate the PoP as necessary, while merely adding a new adapter for each network technology to which the MetaNet interfaces.

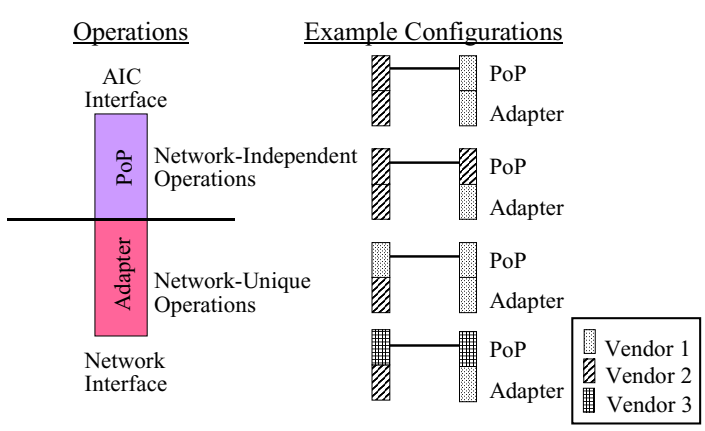

Figure 3 - MetaNet Configurations

Thus the function of the PoP is network independent, while that of the adapter is network dependent. A common interface between PoPs and between a PoP and an adapter will eventually make it possible to integrate PoPs and/or adapters from different vendors as shown in the Figure 3. One vendor could produce all PoPs while any number of other vendors supply adapters designed to control various types of networks. It may be that the network developer may supply an adapter with the network that allows it to be controlled by a PoP.

\subsection{Interfaces}

The PoP receives requests from the AIC for MetaNet channels to be setup, torn down, or modified. The requests include the logical channel source and logical channel destination addresses, the QoS required, and the priority of the request. The MetaNet responds to the AIC, either confirming the setup, or denying the request. There is also a provision for negotiation of the request, such that the MetaNet PoP will provide alternative realizations to the AIC. The QoS is represented as an ntuple of the form: \{capacity, latency, loss rate, jitter, protection, cost,... $\}$. Initially only the first three QoS metrics have been considered.

The PoP receiving the request from the AIC (known as the source $\mathrm{PoP}$ ) partitions the portion of the request to be met locally (if any) by the adapter that it manages. Any PoP may serve as a source PoP for a request. The remainder of the request is forwarded to PoPs, which in turn address that portion of the request under their domain. Each PoP will command its adapter to plan or setup the requested segment of the MetaNet channel that traverses its network. The request can be rejected at either the PoP or adapter level, in which case the PoP can attempt an alternative realization if available, or relay the rejection to the AIC.

The adapter commands the setup, modification, or teardown of the channel segment in its network. This may occur immediately for channels currently required, or may be delayed for channels scheduled in the future. The commanded operation may succeed or fail. In either case, status is provided to the PoP. The source PoP will aggregate the results of the command attempts from each adapter/PoP. If all commands are accepted then the request is feasible and the source PoP informs the AIC. If any of the adapters/PoPs cannot establish a segment of the MetaNet channel, then the request is rejected. Alternatively the source PoP can attempt alternative QoS realizations of the MetaNet channel.

\section{OPERATIONAL CONCEPTS}

A general description of the MetaNet operations has been given above. Here we provide more detail.

The AIC places requests for MetaNet channels with a MetaNet PoP. The request can be made to any PoP and this PoP will act as the source PoP, managing the request and providing a response. PoPs may be located in 
geographically diverse locations with the only provision being IP connectivity with the AIC, another PoP, and the adapter(s) under its purview. Thus, when in operation a given PoP will be managing its MetaNet channels, while other PoPs are managing other MetaNet channels. At the same time each PoP may be invoked by other PoPs to support a segment of a MetaNet channel. The MetaNet channel will realize the logical channel being requested. See Figure 4.

The MetaNet channel will be composed of segments, each of which reside within a single network. The composition of the segments as a whole provide the connectivity from the channel source to the sink (or destination). It is possible for the channel source and sink to reside within one network. In this case, only one segment is required and only one adapter is invoked. The PoP associated with this adapter is also invoked. If this is the source PoP then no other PoP is required. If not, then at least one other PoP acting as the source PoP is invoked.

In general the source and $\operatorname{sink}(\mathrm{s})$ will not reside in the same network and the MetaNet channel must span multiple networks. When this is the case, the MetaNet channel segment in the network of the source must provide a link from the source to a gateway (or border node) to a second network. If the sink is in this network then the segment in the second network will connect the gateway with the sink. Otherwise, the MetaNet channel merely traverses this network to a gateway to another network. This process is iterated until the network in which the sink resides is reached.

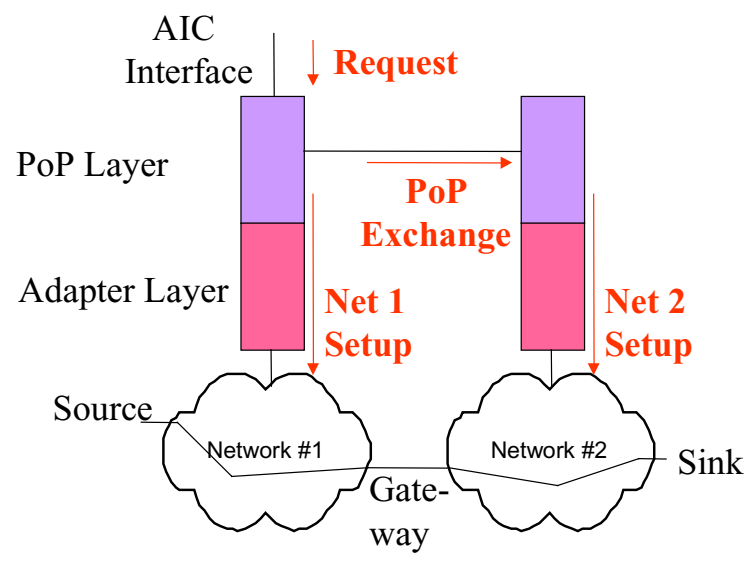

Figure $4-\mathrm{PoP}$ and Adapter Interfaces

The manner in which the MetaNet channel routing over the various networks between the source and $\operatorname{sink}(\mathrm{s})$ is achieved can be accomplished in a number of ways.
One method is by use of a routing table that describes the affiliation of each possible source and sink with a network. Upon identifying the source and sink, a source PoP can access a table to determine the inter-network route to be used. Alternative inter-network routes can be included in the table for redundancy. In this way, the source PoP can immediately determine the PoPs to be invoked to establish a MetaNet channel and the source PoP will communicate with them to direct establishment of the segment in their respective networks. This is the method used in the PoP realized in our project.

Another method of achieving the inter-network routing is by a distributed routing approach. The source PoP determines whether any of the adapters under its control are involved in the MetaNet channel. If so, that portion of the MetaNet channel in this network is noted and the request is modified accordingly and sent via an inter-PoP network to the PoP associated with the next network segment. This PoP then determines the involvement of its own networks and passes a modified request to the $\mathrm{PoP}$ associated with the next segment. Eventually the PoP/adapter controlling the network of the sink is found and a response is sent back along the path of the PoPs to affirm or reject the feasibility of the request. If the request is affirmed by all PoPs in this sequence, then upon reaching the source PoP, the request is confirmed and the MetaNet channel is activated. If any PoP rejects the request, then the source PoP either rejects the request as a whole, or attempts to find an alternative with modified route or QoS, using the same methods.

\section{QoS BROKERING}

When establishing a MetaNet channel that spans multiple networks, the end-to-end QoS required must be partitioned among the network segments. Each QoS parameter is treated independently in this partitioning. For instance, the latency of a channel is cumulative over the segments. The loss rate (e.g., bit error rate or packet loss) is determined as the product of the inverse loss rates of the segments. Thus, each segment contributes to the end-to-end QoS. The manner in which the QoS is partitioned may take various forms. Two of these are described herein.

The QoS of a multi-segment channel is brokered by the source PoP. In the absence of any specific knowledge of the network, the source PoP can equally partition the QoS among the networks spanned. If particular knowledge of a network indicates a characteristic QoS value may be expected, then the source PoP can partition the QoS accordingly. For instance, a satellite link will incur a 0.25 second round-trip delay when a 
geosynchrounous satellite is used, while a terrestrial network can be expected to incur a lower delay. In this case, the source PoP can allocate a 0.25 second delay to the satellite network and then equally distribute the remaining delay specification among all networks spanned. If the delay specification is less than 0.25 seconds then the satellite network cannot be used and alternative routes must be considered.

A second method for partitioning the end-to-end QoS is to employ a distributed approach similar to the distributed route discovery among PoPs. Here the source PoP will, upon receipt of a request, allocate a portion of the end-to-end QoS to the network under its control. The source PoP will then decrement each QoS parameter and pass the modified QoS specification to the next $\mathrm{PoP}$ in the route. The next PoP will then allocate a portion of the remaining QoS to its network. This goes on in turn until the destination is reached or until the QoS is entirely consumed. In the later case, the request will crank-back along the path by which it came, so that the QoS consumed by each network can be modified, with the expectation that adequate QoS will survive to the final $\mathrm{PoP}$ in the route. This approach has a deficiency in that there is no guarantee that the final PoP will ever obtain a realizable QoS specification.

\section{SUMMARY}

An architecture for QoS-based channel setup and routing has been described. It provides a common interface for users requesting services over multiple disparate communications networks. Trade-offs in internetworking routing and QoS brokering have been identified. This MetaNet architecture provides a framework and interfaces for the control and management of DoD information flows providing increased resource utilization and the elimination of stove-pipes. A limited prototype has been developed with interfaces to DoD EHF Satcom and ATM networks to explore some of these concepts.

\section{ACKNOWLEDGEMENTS}

Natarajan Narayanan at Telcordia Technologies motivated many of the topics herein in his own MetaNet project.

Joe Rockmore provided insight as the leader of the AICE Functional Architecture Control Board.

\section{REFERENCES}

[1] AICE DARPA Program, "Agile Information Control Environment, proposers information package (PIP)," BAA 98-26, May 1998.
[2] P. Dharwadkar, H. J. Siegal, and E. K. P. Chong, $A$ study of Dynamic Bandwidth Allocation with Preemption and Degradation for Prioritized Requests, Technical Report, TR-ECE 00-9, School of Electrical and Computer Engineering, Purdue University, July 2000.

[3] A. D, Naik, H. J. Siegal, and E. K. P. Chong, Dynamic Bandwidth Allocation for Requests with Classes and Priorities in Preemptive Distributed Networks, Technical Report, TR-ECE 00-10, School of Electrical and Computer Engineering, Purdue University, July 2000.

[4] J. M. Park, U. R. Savagaonkar, E. K. P. Chong, H. J. Siegal, and S. D. Jones, Efficient resource allocation for QoS channels in MF-TDMA satellite systems, Milcom 2000. 